\title{
Prevalência de manifestações oculares em 198 pacientes com artrite reumatóide: um estudo retrospectivo
}

\author{
Prevalence of ocular manifestation in 198 patients \\ with rheumatoid arthritis: a restrospective study
}

Raphael Guimarães Bettero ${ }^{1}$ Ricardo Faraco Martinez Cebrian² Thelma Larocca Skare ${ }^{3}$
Serviço de Reumatologia - Hospital Universitário Evangélico de Curitiba - Faculdade Evangélica do Paraná FEPAR - Curitiba (PR) - Brasil.

Acadêmico de Medicina da Faculdade Evangélica do Paraná - FEPAR - Curitiba (PR) - Brasil

Acadêmico de Medicina da FEPAR - Curitiba (PR) Brasil.

${ }^{3}$ Chefe da Disciplina de Reumatologia da FEPAR - Curitiba (PR) - Brasil.

Endereço para correspondência: Thelma L. Skare. Rua João Alencar Guimarães, 796 - Curitiba (PR)

CEP $80310-420$

E-mail: tskare@onda.com.br

Recebido para publicação em 29.05.2007

Última versão recebida em 03.08 .2007

Aprovação em 20.08.2007

Nota Editorial: Depois de concluída a análise do artigo sob sigilo editorial e com a anuência do Dr. Moysés Eduardo Zajdenweber sobre a divulgação de seu nome como revisor, agradecemos sua participação neste processo.

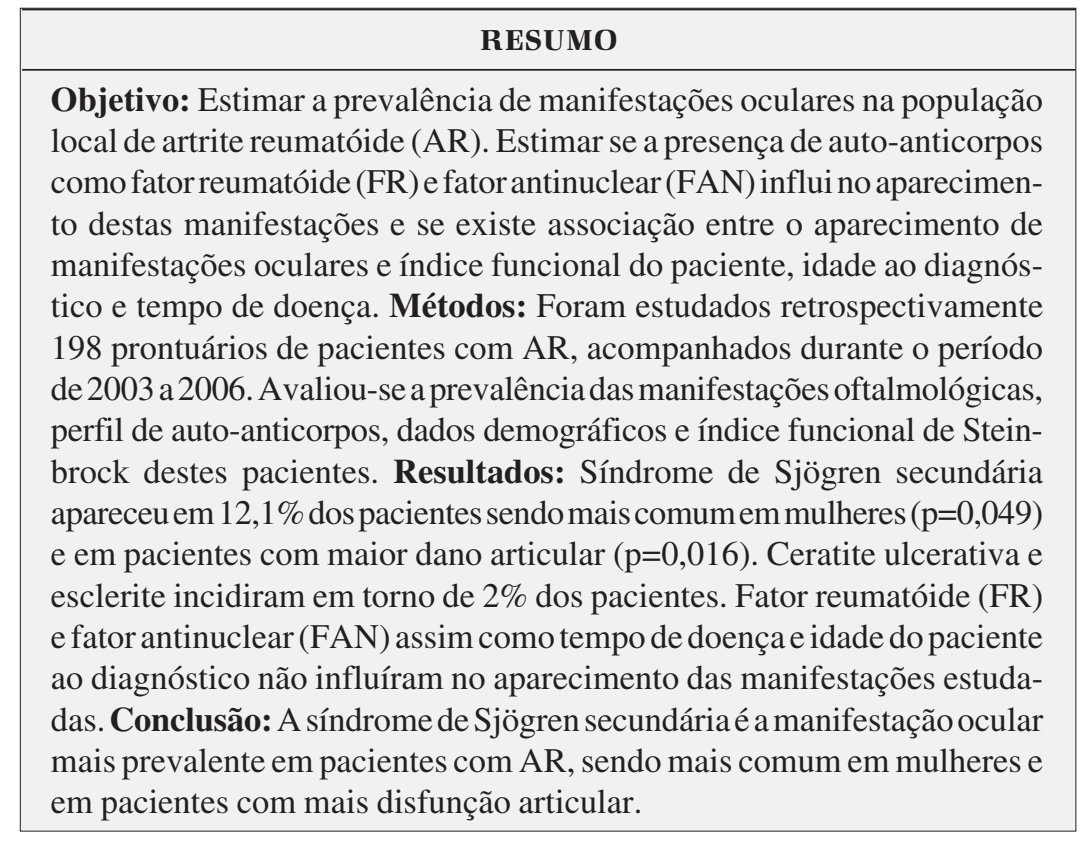

Descritores: Artrite reumatóide/complicações; Oftalmopatias/epidemiologia; Ceratoconjuntivite seca; Síndrome de Sjögren; Anticorpos antinucleares

\section{INTRODUÇÃO}

A artrite reumatóide (AR) é uma doença crônica resultante da presença de processo inflamatório que acomete de forma simétrica todas as articulações sinoviais, principalmente as articulações periféricas de mãos e punhos $^{(1)}$. As manifestações clínicas podem ser persistentes, levando ao aparecimento de deformidades e incapacidades funcionais importantes. Várias manifestações sistêmicas como fadiga, febre baixa, anemia e elevação dos reagentes de fase aguda, como a proteína $\mathrm{C}$ reativa e a velocidade de hemossedimentação, também estão presentes ${ }^{(2)}$.

A AR é uma enfermidade comum. Acomete cerca de $1 \%$ da população adulta mundial $^{(3)}$; sua incidência anual é calculada em 0,1 a 0,2/1000 homens e 0,2 a $0,4 / 1000$ mulheres $^{(4)}$.

A AR, ao contrário do que se pensava até algum há tempo, é uma doença grave que encurta a sobrevida de seus portadores e gera um impacto significativo na qualidade de vida dos indivíduos afetados ${ }^{(1)}$. Nela, o processo inflamatório não está restrito às articulações. Coração, pulmão, músculos e nervos podem ser afetados ${ }^{(2)}$. O olho também está envolvido na forma de 
esclerite, episclerite, ceratite esclerosante, ceratite estromal aguda, ceratoconjuntivite seca secundária, úlcera periférica e "melting" da córnea. A AR é considerada a doença do colágeno que mais comumente afeta a córnea periférica ${ }^{(5)}$.

Sendo essa uma enfermidade na qual aspectos genéticos são importantes, (principalmente a presença de HLAs DR4 e DR1 $)^{(2,6)}$ e na qual o perfil dos auto-anticorpos presente, como fator reumatóide (FR) e fator antinuclear (FAN), influi na gravidade das manifestações apresentadas ${ }^{(7-8)}$, depreende-se que exista variabilidade populacional nos achados articulares e extra-articulares.

O presente estudo procura conhecer a prevalência das diferentes manifestações oftalmológicas na população local de $\mathrm{AR}$, verificar a influência de auto-anticorpos como FR e FAN no seu aparecimento, assim como idade de diagnóstico e tempo de doença. Pretende, também, verificar se as diferentes manifestações oftalmológicas estão associadas com a gravidade da doença articular medida pelo índice funcional de Steinbrock.

\section{MÉTODOS}

Este estudo foi aprovado pelo Comitê de Ética em Pesquisa da Sociedade Evangélica Beneficente de Curitiba sob protocolo 1174/06, em 9 de março de 2006.

Trata-se de um estudo retrospectivo no qual foram revistos 198 prontuários de pacientes com diagnóstico de AR, segundo os Critérios Classificatórios do Colégio Americano de Reumatologia ${ }^{(9)}$ (Tabela 1), que compareceram ao Ambulatório de Reumatologia do Hospital Universitário Evangélico de Curitiba no período de dezembro de 2003 a fevereiro de 2006.

As informações foram alocadas em uma tabela que avaliava a idade do paciente, sexo, tempo de diagnóstico da doença, queixa de secura ocular, presença de ceratoconjuntivite seca, Sjögren secundário, esclerite, episclerite, ceratite ulcerativa, títulos de exames laboratoriais de FR e FAN, e o índice funcional de Steinbrock durante a constatação da possível manifestação oftalmológica.

\section{Tabela 1. Critérios do Colégio Americano de Reumatologia para} classificação dos pacientes com artrite reumatóide

1. Artrite de 3 ou mais articulações (vistas pelo médico e com envolvimento simultâneo);

2. Artrite de articulações da mão (punho e/ou metacarpofalangianas);

3. Artrite simétrica;

4. Rigidez matinal;

5. Presença do fator reumatóide;

6. Nódulos reumatóides;

7. Alterações radiológicas sugestivas da artrite reumatóide. Os critérios de 1 a 4 devem ter uma duração mínima de 6 semanas. Diz-se que um paciente tem artrite reumatóide quando satisfaz 4 dos 7 critérios.

Fonte: Pincus T, Callahan LF. How many types of patients meet classification criteria for rheumatoid arthritis? J Rheumatol. 1994;21(8):1385-9
A queixa de secura ocular foi valorizada quando o paciente apresentava as seguintes queixas: (a) sensação de olho seco por mais de 3 meses; (b) sensação recorrente de areia nos olhos; (c) uso de colírio lubrificante por mais do que 3 vezes/ $\mathrm{dia}^{(10-11)}$. Foi considerado positivo para ceratoconjuntivite seca aquele paciente que apresentou pelo menos um dos seguintes testes alterados: tempo de ruptura do filme lacrimal (BUT; valor normal considerado de até 10 segundos), teste de rosa bengala positivo (valor normal considerado de até 4); e teste de Schirmer diminuído (valor normal considerado acima de $10 \mathrm{~mm}$ ). Para diagnóstico de síndrome de Sjögren utilizaram-se os Critérios Europeus para síndrome de Sjögren ${ }^{(10-11)}$ (Tabela 2).

As demais manifestações oftalmológicas foram consideradas positivas quando atestadas por laudos emitidos pelo Serviço de Oftalmologia do HUEC.

O índice de Steinbrock é um índice funcional global no qual o paciente de AR é classificado de 1 a 4 de acordo com a capacidade de realização de tarefas diárias ${ }^{(2)}$. Para maiores detalhes consultar a tabela 3 .

Os dados coletados foram estudados por tabelas de freqüência e contingência. Foram usados os testes de Mann Whitney, Fisher e Qui-quadrado conforme a variável em estudo, com ajuda do software GraphPad Prism versão 4.0. O nível de significância adotado foi de $5 \%$.

\section{Tabela 2. Critérios Europeus para síndrome de Sjögren}

1. Sintomas oculares (por pelo menos 3 meses);

2. Sintomas orais (por pelo menos 3 meses);

3. Evidência de ceratoconjuntivite seca ao exame (pelos testes de Schirmer, Rosa Bengala e/ou tempo de ruptura lacrimal);

4. Sialoadenite focal (provada por biópsia de glândula salivar menor - na qual se vê pelo menos um grupo de 50 linfócitos ou 1 foco em $4 \mathrm{~mm}^{2}$ );

5. Evidência instrumental de envolvimento de glândula salivar (visto pela medida do fluxo salivar não estimulado menor que $1,5 \mathrm{ml}$ em 15 minutos ou, sialografia e/ou cintilografia de parótida);

6. Presença de auto-anticorpos (fator antinuclear, fator reumatóide, Anti-Ro, Anti-La).

São necessários 4 dos 6 itens para o diagnóstico de síndrome de Sjögren.

Fonte: Goules A, Masouridi S, Tzioufas AG, loannidis JP, Skopouli FN, Moutsopoulos HM. Clinically significant and biopsy-documented renal involvement in primary Sjögren syndrome. Medicine (Baltimore). 2000; 79(4):241-9

Tabela 3. Índice funcional de Steinbrock para pacientes com artrite reumatóide

Classe 1 - Função normal;

Classe 2 - Paciente apresenta alguma dificuldade para realização das tarefas diárias;

Classe 3 - Só é capaz de cuidar de si próprio;

Classe 4 - Precisa de ajuda com tudo, inclusive para cuidado próprio.

Fonte: Skare TL. Artrite reumatoide. In: Skare TL. Reumatologia: princípios e prática. Rio de Janeiro: Guanabara Koogan; 1999. p.91-105 


\section{RESULTADOS}

Dos 198 pacientes estudados, 29 eram homens e 169 eram mulheres. A idade de diagnóstico da AR variou entre 22 e 83 anos (média de 51,6 1 13,7 anos). O tempo médio de duração da doença foi de $9,4 \pm 7,8$ anos.

No grupo estudado foram encontrados: 59 pacientes $(29,8 \%)$ com queixa de secura ocular. Destes $33(16,1 \%)$ tinham ceratoconjuntivite seca e $24(12,1 \%)$ fechavam diagnóstico para síndrome de Sjögren secundária. Esclerite foi diagnosticada em 4 pacientes $(2,02 \%)$ sendo 3 delas anteriores difusas e uma anterior nodular; episclerite foi encontrada em 1 paciente $(0,5 \%)$ e ceratite ulcerativa marginal em $5(2,54 \%)$. Na figura 1 encontram-se representados os dados relativos à prevalência dos diagnósticos oftalmológicos.

Na tabela 4 encontram-se os dados comparativos de prevalência de achados oculares de acordo com o sexo do paciente.

Os dados coletados sobre conjuntivite não foram passíveis de avaliação pela baixa prevalência das manifestações na população estudada (apenas 1 caso) e, nesse não se conseguiu estabelecer nexo causal com a AR.

A tabela 5 indica a prevalência das manifestações oftalmológicas de acordo com a positividade do fator reumatóide. Dos 198 pacientes, 190 foram pesquisados para o FR. Destes 135 (71\%)

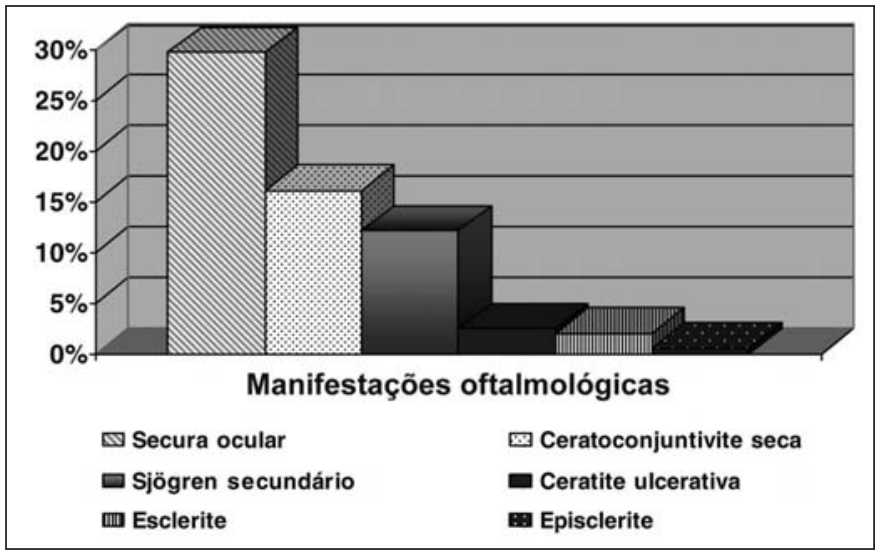

Figura 1 - Prevalência de manifestações oftalmológicas em 198 pacientes com artrite reumatóide eram fator reumatóide positivo e $55(28,9 \%)$ eram fator reumatóide negativos.

A tabela 6 refere-se a 169 pacientes que possuíam pesquisa para FAN e sua correlação com as manifestações oftalmológicas.

A classe funcional de Steinbrock no momento da constatação da manifestação ocular foi obtida em 117 dos pacientes. A tabela 7 mostra as informações relativas à associação entre o índice funcional e manifestações oftalmológicas. As manifestações de esclerite e ceratite ulcerativa não puderam ser analisadas estatisticamente porque só foi achado 1 caso positivo na amostra estudada para essa variável.

A tabela 8 mostra a relação das manifestações oftalmológicas com a idade de diagnóstico obtida em 193 dos pacientes estudados.

Por último, a figura 2 mostra a associação entre manifestações oftalmológicas com o tempo de doença da artrite reumatóide. Esta variável foi obtida em 189 dos pacientes estudados.

Estudando-se a presença das alterações oculares em pacientes com até 10 anos de artrite reumatódie, entre 11 e 20 anos de colagenose e acima de 31 anos encontrou-se $\mathrm{p}=0,12$ $\left(\chi^{2}\right)$ para presença de secura ocular; $\mathrm{p}=0,08\left(\chi^{2}\right)$ para ceratoconjuntivite seca; $\mathrm{p}=0,91\left(\chi^{2}\right)$ para esclerite; $\mathrm{p}=0,1\left(\chi^{2}\right)$ para ceratite ulcerativa e $\mathrm{p}=0,69\left(\chi^{2}\right)$ para Sjögren secundário.

\section{DISCUSS ÃO}

É fundamental enfatizar a associação entre doenças oculares e reumáticas que devem ser bem conhecidas nessas duas especialidades.

\begin{tabular}{|c|c|c|c|}
\hline $\begin{array}{l}\text { Manifestações } \\
\text { oculares }\end{array}$ & $\begin{array}{l}\text { FR positivo } \\
(n=135)\end{array}$ & $\begin{array}{l}\text { FR negativo } \\
\qquad(n=55)\end{array}$ & p \\
\hline Secura ocular & $n=45(33,3 \%)$ & $n=12(21,8 \%)$ & $0,110^{\ddagger}$ \\
\hline Ceratoconjuntivite seca & $n=30(22,2 \%)$ & $n=4 \quad(7,2 \%)$ & $0,002^{\dagger \dagger}$ \\
\hline Sjögren secundário & $n=19(4,0 \%)$ & $n=3 \quad(5,4 \%)$ & $0,130^{\dagger+}$ \\
\hline Esclerite & $n=2 \quad(1,4 \%)$ & $n=1 \quad(1,8 \%)$ & $1,000^{\dagger+}$ \\
\hline Ceratite ulcerativa & $n=4 \quad(2,9 \%)$ & $n=1 \quad(1,8 \%)$ & $1,000^{\dagger+}$ \\
\hline
\end{tabular}

\begin{tabular}{|c|c|c|c|}
\hline $\begin{array}{l}\text { Manifestações } \\
\text { oculares }\end{array}$ & $\begin{array}{l}\text { FAN positivo } \\
\qquad(n=31)\end{array}$ & $\begin{array}{c}\text { FAN negativo } \\
(n=138)\end{array}$ & p \\
\hline Secura ocular & $n=11(35,40 \%)$ & $n=43(31,15 \%)$ & $0,210^{\ddagger}$ \\
\hline Ceratoconjuntivite seca & $n=7 \quad(22,50 \%)$ & $\mathrm{n}=22(15,90 \%)$ & $0,370^{\ddagger}$ \\
\hline Esclerite & $n=1 \quad(3,22 \%)$ & $n=1 \quad(0,72 \%)$ & $0,330^{\ddagger}$ \\
\hline Episclerite & Inavaliável & & \\
\hline Ceratite ulcerativa & 0 & $n=5 \quad(3,62 \%)$ & $0,580^{+t}$ \\
\hline Conjuntivite & Inavaliável & & \\
\hline Sjögren secundário & $\mathrm{n}=6 \quad(19,35 \%)$ & $\mathrm{n}=14(10,10 \%)$ & $0,151^{\ddagger}$ \\
\hline
\end{tabular}




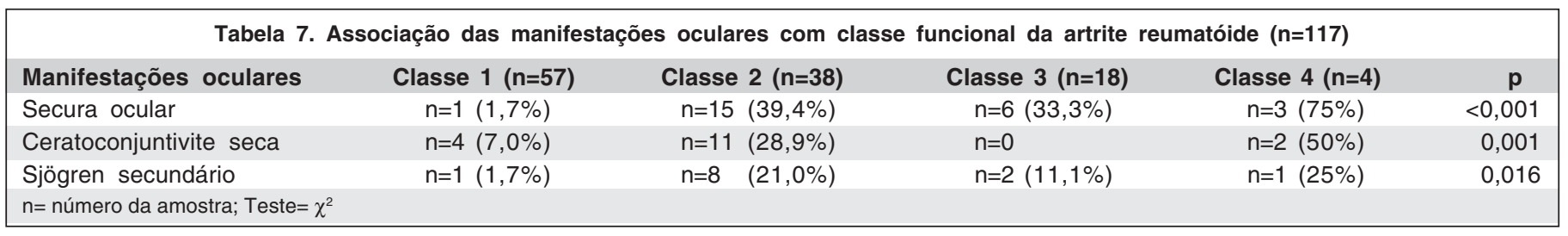

\begin{tabular}{|c|c|c|c|c|c|c|}
\hline Idade de diagnóstico (em anos) & $<30(n=14)$ & $31-40 \quad(n=25)$ & $41-50 \quad(n=50)$ & $51-60 \quad(n=53)$ & $>61 \quad(n=51)$ & $p$ \\
\hline Secura ocular & $n=3 \quad(21,4 \%)$ & $\mathrm{n}=7(28 \%)$ & $\mathrm{n}=18 \quad(36 \%)$ & $\mathrm{n}=11(20,7 \%)$ & $n=19 \quad(37,2 \%)$ & 0,30 \\
\hline Ceratoconjuntivite seca & $n=2(14,2 \%)$ & $\mathrm{n}=2 \quad(8 \%)$ & $n=7 \quad(14 \%)$ & $\mathrm{n}=11(20,7 \%)$ & $n=11 \quad(21,5 \%)$ & 0,54 \\
\hline Esclerite & $\mathrm{n}=0$ & $\mathrm{n}=0$ & $\mathrm{n}=0$ & $n=2 \quad(3,7 \%)$ & $\mathrm{n}=2 \quad(3,9 \%)$ & 0,47 \\
\hline Ceratite ulcerativa & $\mathrm{n}=0$ & $\mathrm{n}=0$ & $(4 \%)$ & $\mathrm{n}=2 \quad(3,7 \%)$ & $n=1 \quad(1,9 \%)$ & 0,77 \\
\hline Sjögren secundário & $\mathrm{n}=2 \quad(14,2 \%)$ & $\mathrm{n}=3(12 \%)$ & $(14 \%)$ & $n=5 \quad(9,4 \%)$ & $n=5 \quad(9,8 \%)$ & 0,93 \\
\hline
\end{tabular}

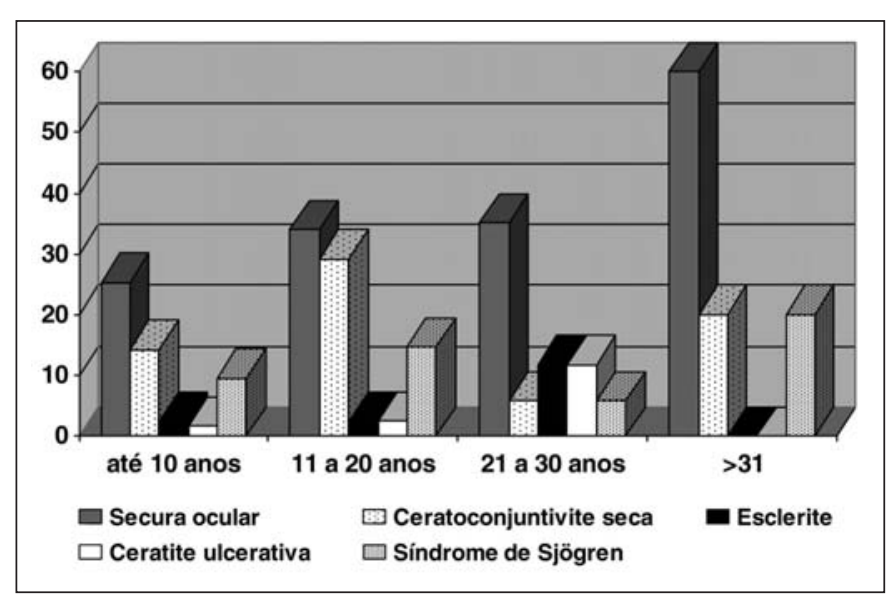

Figura 2 - Manifestações oculares de acordo com o tempo de duração da artrite reumatóide

Queixas de secura ocular e achados de ceratoconjuntivite seca não podem ser atribuídos diretamente a AR uma vez que tais eventos têm etiologia multifatorial, podendo estar relacionados, por exemplo, ao simples envelhecimento ou uso de determinados medicamentos principalmente diuréticos e antidepressivos $^{(12)}$. Levando-se isto em consideração, a manifestação mais comumente encontrada e que pode ser atribuída à AR foi a de síndrome de Sjögren secundária que apareceu em $12,2 \%$ dos pacientes. Todavia, não se pode esquecer que tal síndrome pode iniciar-se pelas manifestações oculares. Por isso, parte dos pacientes que se apresentaram só com queixas e achados de secura ocular podem estar fazendo parte de uma forma incompleta dessa síndrome. A prevalência de síndrome de Sjögren na literatura varia de 11 a $13 \%{ }^{(13)}$, números muito semelhantes aos encontrados na presente pesquisa.

Interessantemente, os achados do presente estudo mostram que o aparecimento de síndrome de Sjögren está associado com classe funcional do paciente, mas não com o tempo da doença. Isso demonstra que aqueles pacientes com uma artrite reumatóide mais grave, mas não os com doença há mais tempo, têm mais chance de desenvolver Sjögren secundário.

Outra associação interessante que pode ser notada foi a maior prevalência de síndrome de Sjögren no sexo feminino. Alguns autores sugerem que estrógenos aumentam o risco de desenvolver sintomas da síndrome seca, uma vez que $96 \%$ da população afetada pela forma primária do Sjögren é mulher ${ }^{(14)}$. A literatura aponta para a maior prevalência de secura ocular em mulheres no climatério recebendo terapia de reposição hormonal, particularmente monoterapia com estrógeno ${ }^{(15)}$. Calcula-se que a cada três anos de uso de terapia de reposição hormonal, aumenta em $15 \%$ o risco de sintomatologia para olho $\operatorname{seco}^{(15)}$.

As demais manifestações oculares foram relativamente raras. Entretanto, se for levado em conta que a AR tem uma prevalência bastante alta na população geral, tais complicações passam a ter maior importância.

No caso da esclerite, a literatura mostra uma prevalência em até $5 \%$ dos pacientes de AR, embora até 33\% dos pacientes que procuram o oftalmologista com esta afecção tenham $\mathrm{AR}^{(16)}$. Essa é uma manifestação grave com potencial de disfunção importante para o órgão e cujo achado está intimamente ligado à atividade inflamatória da AR. Seu aparecimento tem, inclusive, importância prognóstica para o reumatologista, uma vez que sua ocorrência está ligada a uma menor sobrevida destes pacientes $^{(17)}$. Nesta pesquisa, esclerite foi identificada em somente $2 \%$ dos pacientes estudados. A baixa incidência verificada no presente estudo em seu aparecimento talvez se deva à maior agressividade no tratamento da $\mathrm{AR}$ proposta nas últimas décadas.

Sobre as características da ceratite ulcerativa marginal da AR existem poucos estudos. Clewes et al. estudando sete casos, observaram a sua associação com vasculites e presença de FAN $^{(18)}$. Associação com o FAN não pôde ser demonstrada na amostra presente. 
$\mathrm{Na}$ avaliação do paciente com AR, a presença de FR positivo parece indicar uma doença articular e radiológica mais agressiva do que aqueles com sorologia negativa ${ }^{(19-20)}$. Um estudo analisando uma população feminina com AR de diagnóstico recente e com níveis séricos persistentemente positivos para FR mostrou que tal grupo apresentava mais erosões, nódulos, manifestações extra-articulares, diminuição da capacidade funcional e atividade da doença do que as pacientes soronegativas ou soronegativas intermitentes ${ }^{(21)}$. Já a presença do FAN no paciente com AR tem um papel menos claro embora existam autores que associam a sua presença a uma doença de pior evolução ${ }^{(8)}$. Neste trabalho, nenhuma correlação foi possível entre a presença de fator reumatóide ou do fator antinúcleo e as manifestações oculares especificas da AR, inclusive em casos de ceratite ulcerativa.

\section{CONCLUSÃO}

A manifestação ocular especifica para AR mais comumente encontrada é a síndrome de Sjögren secundária, que esteve presente em $12 \%$ dos pacientes estudados, sendo mais frequiente em mulheres e em pacientes com mais disfunção articular. A incidência de ceratite ulcerativa e esclerite foram relativamente raras (em torno de $2 \%$ cada), o que pode estar relacionado a um controle mais agressivo da atividade inflamatória da AR conseguido com medicamentos modernos. FAN e FR assim como tempo de doença e idade ao diagnóstico não influíram no aparecimento das manifestações estudadas.

\section{ABSTRACT}

Purpose: To study the prevalence of eye manifestations in the local rheumatoid arthritis (RA) population, as well as to know if the presence of rheumatoid factor (RF) and antinuclear antibodies (ANA) affect this profile. To study if there is association between eye manifestations and patient's articular functional index, age at diagnosis and illness duration. Methods: We reviewed 198 charts of rheumatoid arthritis patients followed-up from 2003 to 2006. They were studied for: prevalence of ophthalmologic manifestations, autoantibody profile, demographic data and Steinbrock's functional index. Results: Secondary Sjögren's syndrome was seen in $12.1 \%$ of the patients being more common in women $(\mathrm{p}=0.049)$ and in patients with greater articular damage $(\mathrm{p}=0.016)$. Ulcerative keratitis and scleritis appeared in $2 \%$ of patients each. Antinuclear antibodies (ANA) and rheumatoid factor (RF) as well as illness duration and age at diagnosis had no influence in the appearance of eye manifestations. Conclusion: Secondary Sjögren syndrome was the most common ocular manifestation in patients with RA, mainly in women and patients with greater joint damage.
Keywords: Rheumatoid arthritis/complications; Eye diseases/ epidemiology; keratoconjunctivitis sicca; Sjögren's syndrome; Antibodies, antinuclear

\section{REFERÊNCIAS}

1. Matteson EL, Cohen MD. Conn DL. Rheumatoid arthritis: clinical features and systemic involvement. In: Klippel JH, Dieppe PA, editors. Rheumatology. $2^{\text {nd }}$ ed. London: Mosby; 1998. v.1. S-5. p.4-1:8.

2. Skare TL. Artrite reumatoide. In: Skare TL. Reumatologia: princípios e prática. Rio de Janeiro: Guanabara Koogan; 1999. p.91-105.

3. Spector TD. Rheumatoid arthritis. Rheum Dis Clin North Am. 1990;16(3):513-37.

4. Ciconelli, RM, Artrite Reumatóide. In: Sato EI, coordenador. Guia de reumatologia. Barueri: Editora Manole; 2004. p.85-97.

5. Kanski JJ. Doenças da córnea e esclera. In: Kanski JJ. Oftalmologia clínica: uma abordagem sistemática. $5^{\underline{a}}$ ed. Rio de Janeiro: Elsevier; 2004. p.95-156.

6. Nepon GT, Nepom B. Genetics of major histocompatibility comples in rheumatoid arthritis. In: Klippel JH, Dieppe PA, editors. Rheumatology. $2^{\text {nd }}$ ed. London: Mosby; 1998. v. 1. S-5. p.7-1:12.

7. Erhardt CC, Mumford PA, Venables PJ, Maini RN. Factors predicting a poor life prognosis in rheumatoid arthritis: an eight year prospective study. Ann Rheum Dis. 1989;48(1):7-13.

8. Caspi D, Elkayam O, Eisinger M, Vardimon N, Yaron M, Burke M. Clinical significance of low titer anti-nuclear antibodies in early rheumatoid arthritis: implications on the presentation and long-term course of the disease. Rheumatol Int. 2001;20(2):43-7.

9. Pincus T, Callahan LF. How many types of patients meet classification criteria for rheumatoid arthritis? J Rheumatol.1994;21(8):1385-9. Comment in: J Rheumatol. 1995;22(7):1434-5; author reply 1435-6. J Rheumatol. 1995;22(7):1435; author reply 1435-6.

10. Goules A, Masouridi S, Tzioufas AG, Ioannidis JP, Skopouli FN, Moutsopoulos HM. Clinically significant and biopsy-documented renal involvement in primary Sjögren syndrome. Medicine (Baltimore). 2000;79(4):241-9.

11. Fox R. Sjögren's syndrome. In: Kelley WN, Ruddy S, Harris Jr ED, Sledge C. Textbook of rheumatology. $5^{\text {th }}$ ed. Philadelphia: W.B. Saunders; c1997. vol 1. p.955-68.

12. Fox RI. Sjögren's syndrome. Controversies and progress. Clin Lab Med. 1997;17(3):431-44.

13. Bron JA. The red eye in rheumatoid arthritis. In: Klippel JH, Dieppe PA, editors. Rheumatology. $2^{\text {nd }}$ ed. London: Mosby; 1998. v.1. S-4. p.16.12-16.14.

14. Pillemer SR, Matteson EL, Jacobsson LT, Martens PB, Melton LJ $3^{\text {rd }}$, O'Fallon WM, Fox PC. Incidence of physician-diagnosed primary Sjögren syndrome in residents of Olmsted County, Minnesota. Mayo Clin Proc. 2001; 76(6):593-9.

15. Schaumberg DA, Buring JE, Sullivan DA, Dana MR. Hormone replacement therapy and dry eye syndrome. JAMA. 2001;286(17):2114-9. Comment in: JAMA. 2002;287(5):585-6.

16. McGavin DD, Williamson J, Forrester JV, Foulds WS, Buchanan WW, Dick WC, et al. Episcleritis and scleritis. A study of their clinical manifestations and association with rheumatoid arthritis. Br J Ophthalmol. 1976;60(3):192-226.

17. Coles RS. Ocular manifestations of connective tissue disease. Hosp Pract (Off Ed). $1985 ; 20(2): 70-6,78,80$.

18. Clewes AR, Dawson JK, Kaye S, Bucknall RC. Peripheral ulcerative keratitis in rheumatoid arthritis: successful use of intravenous cyclophosphamide and comparison of clinical and serological characteristics. Ann Rheum Dis. 2005; 64(6):961-2.

19. Listing J, Rau R, Müller B, Alten R, Gromnica-Ihle E, Hagemann D, Zink A. HLA-DRB1 genes, rheumatoid factor, and elevated C-reactive protein: independent risk factors of radiographic progression in early rheumatoid arthritis. Berlin Collaborating Rheumatological Study Group. J Rheumatol. 2000;27(9): 2100-9. Comment in: J Rheumatol. 2002;29(12):2662; author reply 2662-3.

20. Lindqvist E, Eberhardt K, Bendtzen K, Heinegård D, Saxne T. Prognostic laboratory markers of joint damage in rheumatoid arthritis. Ann Rheum Dis. 2005;64(2):196-201.

21. Van Zeben D, Hazes JM, Zwinderman AH, Cats A, van der Voort EA, Breedveld FC. Clinical significance of rheumatoid factors in early rheumatoid arthritis: results of a follow up study. Ann Rheum Dis. 1992;51(9):1029-35. 